\title{
A socio-cognitive framework for researching learning with IMM
}

\author{
Tony Fetherston \\ Multimedia and Learning Technologies \\ Edith Cowan University
}

\begin{abstract}
Interactive Multimedia (IMM) with its rich blend of graphics, sound and movies, is often difficult to conceptualise by researchers. In order to study IMM either in its own right or to examine the effects of the use of IMM in fields like education, researchers need to choose a well described theoretical framework to guide their study. A choice often facing researchers is whether to view IMM as a new media requiring its own unique underlying theoretical framework or to rely on existing frameworks from other fields to underpin their studies.
\end{abstract}

In this paper, Gardner's (1993) idea of multiple intelligences is proposed as a suitable candidate for consideration as at least part of a theoretical framework. Initially the role of a theoretical framework in research is described and this is followed by a brief description of Gardner's ideas. The paper concludes with an example of the utility of using multiple intelligences in a theoretical framework to underpin the study of learning with IMM.

Traditionally, learning is viewed as being mediated both socially and cognitively (Shulman, 1986, p17) and in classrooms this view often assumes some link between teacher behaviour and eventual student performance. According to this view, the teacher is considered able to mediate the processes that occur between the input of instruction and learning outcomes. This view of learning, with its roots in behaviourist psychology, can be modified to take into account a more constructivist view of learning by considering that both social and cognitive mediation processes can occur concurrently in students' minds. Interactive multimedia (IMM) can be part of such a simple, high level model by considering well designed IMM as also being able to mediate in the learning process. The amount of mediation can vary from almost none (pure entertainment) to almost all (no teacher intervention). In this way IMM can be viewed as being able to supplement teaching and learning processes that occur in classrooms. Figure 1 below illustrates this position. 


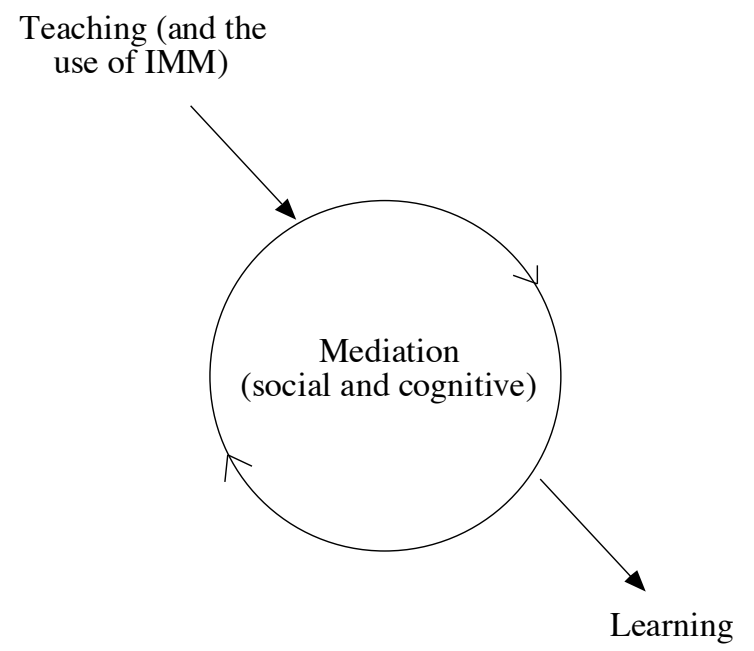

Figure 1: A simple overview of mediation in learning

The overview presented above and in Figure 1 needs further explanation before it can be useful as a theoretical framework. Before it can be further "unpacked" the role of a theoretical framework in research IMM should be discussed.

\section{Theoretical frameworks}

There is growing use of IMM in schools and universities and increasing amounts of IMM delivered through the World Wide Web. Before IMM can be used to best advantage in education, research needs to be conducted that will generate pedagogical guidelines for its use in the various educational contexts. These different contexts can include schools, universities, distance education students, adult learners and training organisations.

At this time there are few, if any, well stated theoretical frameworks to guide the study of learning with interactive multimedia. Studies are often located in constructivism, a framework which is vague, often not clearly stated but fashionable, often without clear links to its theoretical underpinnings or to the kind of constructivism referred to. Studies are sometimes located in other broad domains like positivism or phenomenology which do have clear underpinnings but are so general 
that the study's ability to contribute meaningfully to the theory is severely diminished.

A theoretical framework for studying learning with IMM should have explanatory power relevant to both mediating processes described above and any adequate explanation of learning with IMM should be grounded in both central processes. This means that the framework eventually used probably needs to be able to integrate both a social and cognitive view of learning.

The cognitive view may need to take into account micro-process like the engagement of schemata, the existence of preconceptions and attributions or may concentrate on broader process, suggested by theorists like Piaget (1972), of accomodation and assimilation. It may include general theoretical perpsectives such as the use of mental models (for example Wild, 1996) and situated cognition (Choi \& Hannafin, 1995, as an example in this domain).

The social mediation could well encompass a social constructivist approach like that of Vygotsky (1983). His ideas of mediation in learning, the provision of psychological tools, the zone of proximal development and the general notion of learners coming to know the dominant culture all seem to have prima facie relevance to IMM. Other possible candidates for this section could be the work of Kelly (1955) and his Personal Construct Psychology, which provides a well explained theory of constructivist learning. This was used by Fetherston (1997) to generate hypotheses to underpin a learning approach that could be usefully applied to the use of IMM in classrooms.

\section{Role of a theoretical framework}

A theoretical framework is necessary in studying learning with IMM, for many reasons. The main reason is that the readers of the results of the research need to know "from where the researcher is coming. A well delineated framework should convey to the reader some idea of the political, social, cultural and historical context in which the study is set. A good framework can also explicitly articulate the underlying approach to the analysis of the collected data. Simply, the readers of the research want to know through what eyes the data were viewed. For example, does the researcher have a positivistic view of knowledge or is it interpretivist? 
The framework can assist the researcher with the classification and the identification of patterns in the data if this is desired and matches the research approach.

The term theoretical framework is often used synonymously with the term conceptual framework. Although very similar, one clear difference between the two is that "theoretical framework" should be used when the existing literature or conceptual framework cannot provide a clear understanding of the phenomenon about to be studied. This means that often alternative models may be necessary and these can be used to examine existing assumptions thus becoming a theoretical framework. Frameworks should always have a clearly delineated boundary.

A well stated theoretical framework is essential when studying learning with IMM as it is difficult to define a coherent body of knowledge that could be accepted as underpinning all studies. The study of IMM draws upon many fields including psychology, sociology, science, semiotics and education to name but a few. Consequently, it is important for research in this area to allude clearly to the fields chosen to underpin the study and to give direction as to how results should be interpreted.

Whatever theoretical framework stance is adopted, it must be grounded in critical, scholarly work to give reassurance that the research is valid and reliable. Additionally the study undertaken needs to generate results that can contribute to the theory on which the study is based and also on the theoretical framework.

Taking all the above into account, Gardner's (1993) theory of multiple intelligences appears to be able to make a useful contribution as one component of a theoretical framework for the study of learning and IMM. It is worthy of consideration for inclusion in part of the cognitive mediation section of the simple model proposed above. The theory is briefly summarised below and then an example of its explanatory power is presented.

\section{The theory}

Gardner (1993, pxiv) defined intelligence as "the ability to solve problems, or to create problems, that are valued in one or more cultural settings". He located his view of intelligence amongst lay, psychometric approaches, and pluralisation and hierarchisation theories of intelligence. Using this definition, he empirically derived a set of 
intelligences from literally hundreds of studies using eight distinct criteria for inclusion as an intelligence. These criteria for inclusion were based mainly on biological and anthropological evidence (Gardner. 1993) and were:

Potential isolation by brain damage;

An identifiable core operation or set of operations;

A distinctive developmental history, along with a definable set of expert "end-state" performances;

An evolutionary history and evolutionary plausibility;

Support from experimental psychological tasks;

Support from psychometric findings; and

Susceptibility to encoding in a symbolic system.

Derivation of the distinct intelligences followed a review of existing theories, and in most cases strong critiques, of the then existing approaches to intelligence. This wide ranging review and critique examined phrenology, the psychometric approach, Piagetian views, cognitive views and human systems approaches. General criticisms that arose from all these studies were that most of these views of intelligence focussed on logical or linguistic problem solving, all of them ignored the influence of biology, all did not satisfactorily explain creativity or originality and all were insensitive to the range of roles that exist in human society.

Candidates rejected for inclusion as an intelligence, included intuition, common sense and shrewdness. Gardner (1993) regarded intelligences as being broader than just computational capacities but narrower than a general ability. Like Gall in the 1790s he proposed that we have no general intelligence, that we posses memories that are specific to each of our intelligences and that we think differently using each of the separate intelligences.

The intelligences finally proposed were:

- Linguistic;

- Musical;

- Logical-mathematical; 
- Spatial;

- Bodily-Kinaesthetic;

- Interpersonal;

- Intrapersonal;

and latterly it appears that an eighth intelligence is likely to be added to the list, that of the Naturalist.

\section{Applications of the framework}

The theory of multiple intelligences is well argued from an empirical base and consequently fulfills one criterion for use as at least part of a theoretical framework. However, a more interesting criterion is its explanatory power. Does it have sufficient theoretical substance to assist us with our study of learning with IMM and to let us explain key constructs? In the following, I would like to demonstrate its utility.

IMM can assist learning by providing an interesting and useful alternative to traditional lessons. IMM can provide a rich learning experience for students with its ability to represent and simulate many experiences that are either not readily available or dangerous. However the construct richness is a multi faceted construct. It can mean to:

- provide all the common media on a single platform;

- be able to select and combine these media to produce desired presentations;

- be able to provide much wider choices of learning paths; or

- be able to individualise instruction.

Richness depends mostly upon the variety of symbol systems available in usual IMM product. This variety of different symbol systems almost always enables access to learning by more learners than the use of traditional print materials. But why does this richness of symbol systems, prove interesting to learners and provide them with learning alternatives? An explanation is provided by the use of multiple intelligences.

One of Gardner's (1993) criteria for including an intelligence in his list was the susceptibility of the intelligence to encoding in a symbolic system. Because of this encoding, it can be proposed that the variety of symbol systems often used in IMM engages several of a learner's 
separate intelligences. Taking this argument further, an IMM product could be viewed in terms of these three criteria:

- the number of separate intelligences it engages;

- the extent that each is engaged; and

- how well content is able to be accessed through each separate intelligence (working in conjunction with the corresponding symbol system of the IMM product).

The above three points can provide a framework for evaluation of a product in cognitive terms. Such evaluation would be most useful at the instructional design stage of product development. Decisions could be made at this stage of development as to which symbol systems and consequently, intelligences to be engaged by the product. Clearly, the challenge lies at the design stage of a product to be able to design a product that can successfully cater for these different intelligences.

Closely related to engagement is motivation. IMM is commonly assumed to motivate the learner automatically simply through the use and integration of the media involved. This degree of intrinsic motivation can now be teased out and related to the seven intelligences that is the degree of intrinsic motivation will rely to a large extent on engagement of the seven intelligences.

These intelligences can be engaged to different extents by different activities that commonly take place in a school.

Table 1: How different intelligences might be engaged by different activities at school.

\begin{tabular}{|c|c|c|c|c|c|c|c|c|}
\hline \begin{tabular}{|l} 
Intelligence \\
Learning activity
\end{tabular} & Ling. & Mus & $\mathrm{L}$ & Sp. & Bod & IP & $\operatorname{IrP}$ & Nat \\
\hline Reading from a book & $\boldsymbol{V}$ & & $\boldsymbol{V}$ & & & $\boldsymbol{V}$ & $\sigma$ & \\
\hline Prac work in science & & & $\boldsymbol{v}$ & $\boldsymbol{V}$ & $\boldsymbol{V}$ & $\boldsymbol{V}$ & & \\
\hline Excursion & & & & 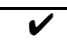 & $\boldsymbol{v}$ & $\boldsymbol{V}$ & & $\boldsymbol{V}$ \\
\hline Cooperative learning & & & $\sqrt{\sigma}$ & & & $\bar{v}$ & $\sigma$ & \\
\hline $\begin{array}{l}\text { Usual transmissive } \\
\text { lesson in school }\end{array}$ & $\bar{v}$ & & $\sigma$ & & & & & \\
\hline
\end{tabular}


Table 1 shows that reading might engage linguistic, logical mathematical, interpersonal and intrapersonal intelligences. A transmissive lesson where students listen to a teacher's explanation and then just copy down some notes might only engage linguistic and logical-mathematical intelligences or even just the linguistic intelligence. This table makes clear that IMM is more engaging to most students than usual lessons because of its ability to engage more intelligences.

Seeing IMM product from a multiple intelligence view helps us to understand the individual nature of the perception of IMM and the often idiosyncratic nature of the effect of IMM on individuals and their learning. This is because this approach recognises that individuals differ in "the forms of these representations, their relative strengths, and the ways in which (and ease with which) these representations can be changed" (Veenema and Gardner, 1996, p70). In other words different individuals prefer to use their different intelligences to different extents.

Of further interest is the application of the theory of multiple intelligences to the general area of useability and useability testing. Another dimension can be added to this field by considering useability in terms of the degree to which users can use or chose to use or have to use their separate intelligences. Similarly, interface design can also be considered in terms of the employment of separate intelligences by the user.

\section{Conclusion}

The use of Gardner's (1993) theory in a socio-cognitive theoretical framework to underpin studies of IMM is worthy of consideration. This is because it is: well explicated; capable of considerable explanatory power; offers an alternative view to usual cognitive mediation approaches; and can easily be integrated with social mediation aspects to form a cohesive framework.

\section{References}

Vygotsky, L. S. (1986). Thought and language. (Ed. A. Kozulin). Cambridge, MA: MIT Press.

Piaget, J. (1972). Intellectual evolution from adolescence to adulthood. Human Development, 15, 1-12. 
Veenema, S \& Gardner, H. (1996). Multimedia and multiple intelligences. The American Prospect, 29, 69-75.

Gardner, H. (1993). Frames of Mind (2nd edition). London: Farmer Press.

Fetherston. T. (1997). The derivation of a learning approach based on personal construct psychology. International Journal of Science Education, 19(7), 801-821.

Kelly, G. A. (1955). The Psychology of Personal Constructs. New York: Norton.

Choi, J-I. and Hannafin, M. (1995). Situated cognition and learning environments: Roles, structures, and implications for design. Educational Technology Research and Development, 43(2), 53-69.

Wild, M. (1996) Mental models and computer modelling. Journal of Computer Assisted Learning, 12(1), 10-21.

Shulman, L. S. (1986). Paradigms and research programs in the study of teaching: a contemporary perspective. In M. Wittrock (Ed). Handbook of Research in Teaching. New York: MacMillan. 